\title{
ELEMENTOS PARA UNA PROSPECTIVA DE LA UNIVERSIDAD COLOMBIANA
}

Por

Alhim Adonaí Vera Silva*

\section{RESUMEN}

En el presente artículo se analizan las relaciones entre las normas concebidas desde la burocracia de los partidos tradicionales, que amarran el futuro de las universidades a sus intereses particulares representados en los Consejos Superiores, a través de por lo menos dos figuras: la discrecionalidad y la delegabilidad, frente a los retos de la sociedad del conocimiento que invita a construir por consenso los escenarios de los proyectos de universidades del futuro donde las normas son soportes que garantizan la transformación colectiva. El argumento central de esta tesis considera que, primero, se concibe el nuevo proyecto de universidad del futuro y sus escenarios; segundo, se concertan los escenarios futuribles (1) de la universidad en una Asamblea general Universitaria, (2) (como el organismo de mayor poder democrático (3) universitario); tercero, se convierte en normas las decisiones asumidas por la Asamblea General Universitaria; cuarto se establecen los requisitos y compromisos económicos, científicos, tecnológicos y académico administrativos para la transformación y quinto se inicia el proceso de transformación universitaria con el concurso de los distintos actores políticos, académicos, culturales, sociales y productivos. Como estudio de caso se abordará la Universidad Surcolombiana.

\section{PALABRAS CLAVES}

Prospectiva, futurible, Burocracia, HCSU (Honorable Consejo Superior Universitario), Discrecionalidad, Delegabilidad.

*Doctor en Educación, Línea Educación Superior Internacional y Comparada: Universidad Autónoma del Estado de Morelos, México. Magister en Investigación y tecnología Educativa: Universidad Pontificia Javeriana, Colombia. Licenciado en Ciencias de la Educación con especialidad en Psicopedagogía: Universidad de Pamplona. Colombia. Profesor titular Universidad Surcolombiana desde 1983. 
La reflexión en torno al papel de los actores universitarios en la transformación de la universidad Surcolombiana, tiene que ver con la, relación entre la visión prospectiva (4) de la universidad, las normas que la regulan y el compromiso cientifico, político, económico, social y cultural que deben asumir éstos actores universitarios para su transformación.

No ha nacido el nuevo proyecto de Universidad Surcolombiana del futuro y las disquisiciones de la universidad giran en cómo construirle su jaula normativa, su corral de articulos; qué ingeniosos somos para amarrar, controlar, inmovilizar lo nuevo, que la universidad no se escape a nuestra voluntad y dominio.

Lo nuevo nos produce inmovilidad teórica que solucionamos con la norma burocrática, con la magia reguladora de lo escrito; qué tantos palitos debe tener la jaula inmovilizadora del futuro, unos cuantos artículos por aquí, otros por allá, un paragrafito para que la discrecionalidad se haga visible; unos incisos para la delegabilidad mutante; unas trancas mas allá para los académicos audaces que les da por pasar la frontera legal, unos mataburros en aquella curva que permita eliminar aspectos indeseables competitivos, qué tal les parece un articulado sobre la imagen simbólica del rectorable, unos requisitos escritos que nos eviten disfrutar su sabiduria. Qué hábiles somos nosotros para conservar, lo viejo por lo viejo o por su potencial de prebendas y que frágiles somos para abordar la complejidad del futuro a través de la visión de sus escenarios.

En el nuevo escenario de la sociedad del conocimiento, la norma tradicional que funcionó para consolidar el desarrollo de la economía de los poderosos en la sociedad esclavista griega, en la sociedad romana discriminatoria, en la oscurantista feudal, o en la capitalista salvaje está en crisis por su incapacidad de regular las relaciones de poder en la nueva sociedad del conocimiento. La fragilidad de la dimensión normativa tradicional referida a los Estados actuales está descrita en una bella paradoja de Carlos Tünermann (5) cuando afirma: «El Estado actual es demasiado grande para las pequeñas cosas y demasiado pequeño para las grandes cosas", estamos frente a un Estado incapaz de regular el futuro de la universidad, desde el discurso normativo, ni menos aum, proyectarlo, a través de la discrecionalidado o de la delegabilidad que le han otorgado a su órgano de poder: «El Consejo Superior Universiterio (6)».

La tradicional composición de los órganos de poder de una organización universitaria pưblica regulado por la norma, tiene un signifie do oculto que la relaciona con las mismas concepciones de poder y de organización que manejan las burocracias (7) en sus partidos tradicionales y en los órganos del Estado bajo dos figuras: la "delegabilidad (8)" y la "discrecionalidad (9)" Las formas tradicionales de la articulación de los partidos en la toma de decisiones tienen relaciones con estas dos figuras que adquieren su cuerpo en el instante del voto. Con relación a la "delegabilidad" los delegados los hay de tantas formas: edad, categoria, tinte, atractivo, fuerza y pedigrí, dependiendo del poder con que ha sido ungido el delegado para que lo juegue en la mesa, o fuera de la mesa, o debajo de la mesa; incluso es común, encontrar delegado, del delegado, pendiendo de las decisiones que deba tomar el consejo ese día. Existen además, formas de usar las figuras de los delegados que se expresan en funciones sui géneris como: delegado emergente, delegado suplente, delegado incógnito, delegado encapsulado, delegado silencioso; algunos, transforman las leyes de la materia y adquieren el don de la ubicuidad. Lo fascinante de este fenómeno de la delegabilidad, es que el delegado puede aparecer y desaparecer por arte de magia en el momento de las decisiones. 
Con relación a la discrecionalidad, los consejos superan las mismas concepciones deprimentes que manejan las burocracias tradicionales de los partidos, parece que no es vital el capital cultural palabras de Bourdieu de los actores universitarios para construir el nuevo ethos académico de la universidad; por ello, quizás se explica, la fragilidad de ciertas paridades numéricas o ciertos coeficientes matemáticos que dan «legitimidad" o "deslegitimidad» a las decisiones a través de unos numerillos, a los cuales se les puede estirar o encoger el pescuezo, cuando por ejemplo, de elegir a un rector se trata; lo importante es que el elegido quepa dentro del hueco normativo que la discrecionalidad y la delegabilidad que el "Consejo" le ha propiciado.

Desde esta perspectiva es fundamental estudiar con rigurosidad cientifica, el grado de las contradicciones que el manejo del poder ha generado como símbolo de intromisión de los partidos politicos tradicionales y del Estado, en el fenómeno llamado universidad, en nuestro caso, Universidad Surcolombiana y sus efectos en la construcción del futuro de la universidad. La arbitrariedad que vulnera la «autonomía» de las universidades y fortifica a los Estrados Judiciales que deslegitiman en horas, lo que le costó a la universidad construir en siglos.

En este orden de ideas los «Honorables Miembros del Consejo Universitario (HCSU) deber ser las personas más idóneas, éticas, morales y sabias de la sociedad del conocimiento internacional, nacional y huilense que nos representan; no sólo el porte de pergaminos académicos muestra el sendero caminado por el novicio a miembro del HCSU; sino que su figura académica, su respetabilidad social es reconocida por las comunidades cientificas y por tanto merecen nuestro respeto, admiración y liderazgo.
Se imaginan por un instante en el HCSU de la Universidad Surcolombiana reunidas en diálogo creativo y prospectivo las mentes más preclaras, mas lúcidas, más brillantes, más connotadas del pais y de la región creando, proponiendo, construyendo, boceteando, gestionando, generando ambientes y escenarios para la maduración de las ideas, propiciando las innovaciones, las estrategias de trabajo, la modernización de la infraestructura científicotecnológica, gestionado fuentes de financiación y cooperación regional, nacional e internacional para los proyectos de la universidad del futuro. Un encuentro del saber abierto a los medios de comunicación, en transmisión directa televisada e interactiva, como ya lo hacen el Congreso de la republica, el Senado y la misma Cámara de Representantes, con el rostro en alto ante la sociedad y los estamentos que representan. Este fenómeno no sólo se da en Colombia, sino en la mayoria de países del mundo democrático. Quizás sea cierto la afirmación que ronda los pasillos de la desesperanza intelectual: La universidad es una democracia sin demócratas, que forma demócratas.

El recinto del HCSU debe ser el espacio donde se valoran y anticipan las múltiples tendencias del conocimiento y de los impactos en la formación humanística, científica, tecnológica y artística de las actuales y próximas generaciones. Existen acercamientos entre las fronteras de las ciencias, las tecnologias y las innovaciones dando un nuevo sentido a la transversalidad del conocimiento; nuevas profesiones brotan de estas alianzas científicas e inéditas tecnologias, con profundizaciones disciplinares, interdisciplinares, multidisciplinares y transdiciplinares; con rupturas, fases creativas, y fases destructivas (10) que marcan una nueva forma de percibir, interpretar y transformar el universo antes desconocido y hoy en proceso de inserción en la cotidianidad de la investigación científica de frontera. 


\section{Elementos para una prospectiva de la universidad Colombiana.}

Es esencial, vislumbrar los escenarios de la universidad del futuro, en una época de transición, entre lo mecánico y lo vital, en un escenario conflictivo planetario, y la añoranza de una comunidad pastoril y donde la sociedad del conocimiento aún no se consolida como cuerpo capaz de convertirse en paradigma. Sin embargo, avizoramos la complejidad conceptual que deberán manejar las nuevas generaciones, mientras permanecemos atarzanados a un mendrugo de poder; sentimos pánico por lo que está por nacer, que ha sido gestado en la misma historia del conocimiento; frutos del ingenio humano, materialización de su inteligencia.

El conocimiento que nace, se construye y se de-construye desde las teorias dialécticas a partir del trabajo en todas sus formas, desde las materiales hasta las más intangibles llegará en esta época a convertirse, en sí mismo, en paradigma de transformación o de alienación social. Por ejemplo, la revolución científicotecnológica y la lectura, interpretación, e intervención en la complejidad del genoma humano, sin duda transformará los ciclos vitales del ser humano, las formas de abordar la vida, las relaciones de la industria farmacéutica, generando variados escenarios con nuevos actores, en antes inéditos campos de conocimiento, que harán surgir nuevos conceptos de ser, valor, ética, amor, sociedad y vida, como nunca la humanidad lo habia sentido.

El HCSU deberá propiciar el análisis sobre la desaparición de campos laborales que duraron por siglos: El conocimiento será el factor central del desarrollo de las sociedades, y de sus mayores conflictos, surgirán nuevos ambientes de aprendizaje que transformarán la actual universidad; se pasará de una universidad que enseña a una universidad que aprende; de una universidad que replica a una universidad que investiga, de una universidad que cree a una universidad que crea; de una universidad rigida a una universidad flexible. Será necesario, anticipar la formación en nuevas carreras más cercanas al conocimiento humanistico de las ciencias básicas; asi como del surgimiento de una nueva complejidad en la interpretación de las ciencias sociales y humanas menos occidentalizadas, y más universales e integradas como plantea Emmanuel Walestein.

Es fundamental prever un acercamiento cada vez más profundo entre las ciencias, las tecnologías y el arte, y la articulación de estos avances en una relación paradójica entre la infinita capacidad de manejo de conocimientos y la miniaturización de la complejidad del conocimiento, temas que deberán ser del dominio de los miembros del HCSU, de los actores universitarios, de la sociedad colombiana y latinoamericana para nuestra articulación e ínterfecundación creativas.

Es necesario, que el $\mathrm{HCSU}$ valorare las visiones de las organizaciones responsables de producir conocimiento en la región; la universidad no es una organización solitaria del conocimiento, existen otras organizaciones que también lo producen, por tanto socios en el camino de su transformación y con ellos hay necesidad de realizar alianzas estratégicas para su abordaje cooperativo, quizás lleguemos a elementos comunes en las visiones con otras organizaciones que tienen que ver con la producción de innovaciones, y partes de la visión que determinan su desarrollo organizacional en su especificidad, pero es posible descubrir una nueva visión compartida, si es necesario revisitaremos el futuro en palabras de Juanjo Gabiña (11) de estas organizaciones que llamaremos del conocimiento. Lo anterior implica que la universidad como lider tendrá que impulsar con la mayor brevedad la configuración de una red de universidades del sur colombiano: Tolima, Huila, Caquetá, Putumayo, Cauca y Nariño, articuladas a las 


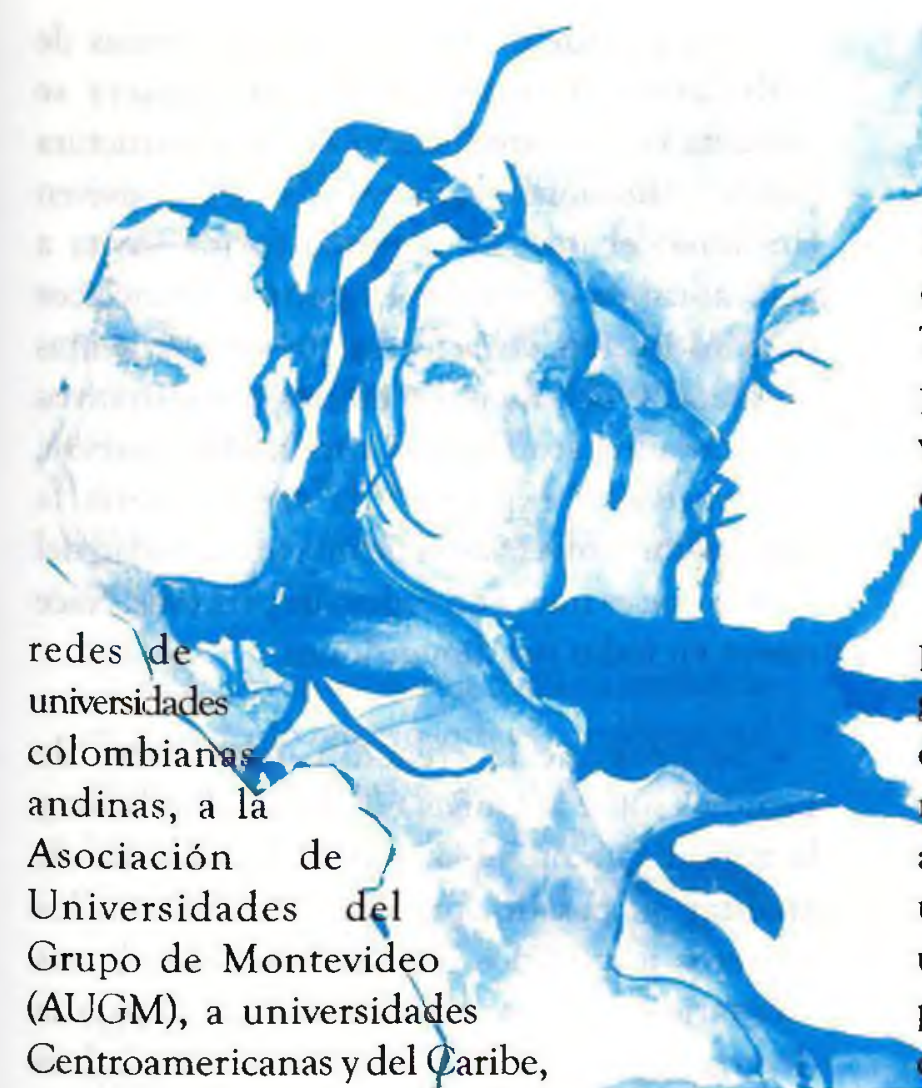

Consejo Superior de Universidades de Centroamérica (CSUCA) a universidades estadounidenses, mexicanas y canadienses, asiáticas, árabes y africanas.

El HCSU debe propiciar los espacios para el estudio de la capacidad científico-tecnológica y humana para que la Universidad Surcolombiana participe en el estudio de la multiriqueza en biodiversidad y cultura del sur colombiano. Existe un macroproyecto planetario sobre la biodiversidad que nos amarra a las Selvas Amazónicas. Es increible que varias universidades del mundo estudian la amazonía y nosotros no tengamos una presencia de investigación significativa a pesar que la universidad del Caquetá, nuestra propia hija, ya pertenece a la red de universidades amazónicas, UNAMAZ. Por cercanía podemos contribuir con nuestros investigadores en el estudio de la complejidad amazónica, además de la riqueza que para los estudios constituye el Puracé, el Macizo Colombiano, el Alto Magdalena y sus corredores biológicos e hídricos; los parques naturales reservas nacionales. Existen escenarios para la antropología, la paleontologia, la arqueologia, la sociología, la astronomia, la historia, el arte y la cultura, combinadas con disciplinas nuevas. El mismo desierto de la Tatacoa es un lugar geoespacial huilense privilegiado con caracteristicas astronómicas de visibilidad muy atractivas para distintos científicos del mundo.

En este escenario existe una crisis interesante, por supuesto creativa, relacionada con el relevo generacional, estamos frente a la oportunidad de entregar a las nuevas generaciones las llaves maestras de los escenarios que serán los adecuados para la transformación de la universidad, el desarrollo histórico de la universidad como constructo teórico que perduró por siglos, está en crisis, no sólo es la crisis de la Universidad Surcolombiana, o de la universidad colombiana; es una crisis planetaria que estremece las estructuras añejas de la normatividad universitaria del derecho, que pretende regular el futuro maniatándolo a sus articulados que niegan la transformación de la universidad. La tensión en la transformación de las universidades parece centrarse en el dilema "construir el futuro de la universidad a partir de las normas o construir el futuro de la universidad a partir de la transformación del conocimiento».

\section{PROPUESTAS}

- Crear los espacios y ambientes para la construcción del nuevo proyecto de universidad para la universidad Surcolombiana, que convoque a todos las actores políticos, académicos, estudiantiles, económicos, sociales y culturales a su construcción, éste proceso implica una transformación en las concepciones curriculares y en las formas organizacionales de la universidad: para lo cual se propone la publicación de las distintas propuestas en un texto a ser analizado por toda la comunidad 
académica y sometido a una Asamblea General Universitaria como máximo organismo de decisión con estrategias consensuadas entre los actores politicos, sociales, culturales, académicos y estudiantiles.

- Construir un compromiso de acción ante una visión institucional y compartidas con todas de las universidades de la región Surcolombiana, que a través de redes articulemos escenarios de aprendizajes, investigación, desarrollo social e innovaciones. La transformación de la universidad que en palabras de Didriksson (12) debe vivir una fase de transición caracterizada por episodios donde los simbolos de la universidad tradicional se mantienen vivos y los símbolos de la nueva universidad productora de conocimientos, anuncian su fuerza naciente. A partir del nuevo proyecto de universidad Surcolombiana, se deben encontrar las alianzas estratégicas correspondientes y desarrollar los pactos que deben ser establecidos a través de relaciones que integren los ingredientes de la nueva sociedad del conocimiento, preferentemente los lineamientos de la última cumbre sobre la educación superior realizada en Paris en 1998.

- Es fundamental pensar en estructuras hibridas en un proceso de transición entre la sociedad industrial y la sociedad del conocimiento para el inicio de la transformación de la universidad, que requerirá una fuerte modernización en su infraestructura cientifico-tecnológica y de formación de talentos humanos desde una visión humanistica. El paso de la transición implica la presencia, aún de los rasgos de la universidad tradicional, pero a su vez, la creación de los escenarios para la nueva universidad. La experiencia en la transformación de universidades demuestra que es un proceso, conflictivo, duro, extenuante, que debe ser acompañado por organismos y expertos nacionales e internacionales en la transformación de las universidades.
- Según Didriksson existen tres formas de transformar la universidad, la primera se sustenta en la introducción de otra estructura paralela autónoma, la nueva y la vieja conviven sin amancebarse. La segunda forma invita a colocar cargas explosivas en puntos estratégicos de la institución, de tal forma que sus estructuras obsoletas se debiliten por efectos demostrativos de unidades académicas totalmente nuevas, innovativas y exitosas y la tercera sería la innovación universitaria de una universidad del futuro que es nueva en todos sus procesos. Nace nueva en todas sus dimensiones.

- Se propone la creación del Centro de Estudios sobre la Universidad Surcolombiana y la región Surcolombiana con el propósito de analizar su pasado, presente y futuro en los aspectos implicados con el desarrollo de sus escenarios. En la actualidad el espacio para la divergencia está congelado, la diversidad es frágil, la heterogeneidad se asocia con decadencia, la denuncia y la sospecha son productoras de poder; ésta sola actitud compromete las nuevas formas de producción del conocimiento.

- Se propone transformar la oficina de relaciones internacionales en una organización académico-cientifica con la capacidad de gestionar proyectos, recursos y estrategias que jalonen la transformación de la universidad Surcolombiana.

\section{NOTAS}

(1) Concepto acuñado por el prospectólogo francés Michael Godet para describir el escenario posible o realizable, entre múltiples futuros, desde el compromiso de los actores.

(2) Los Honorables Consejos Superiores de las universidades mexicanas para abordar las últimas crisis han acudido a la 
figura de las Asambleas Generales Universitarias. Caso de la Universidad Autónoma del Estado de Guerrero, UAGRO. 1999 - 2001. Consultar resolutivos del Consejo General Universitario Acapulco, 2001. Esta universidad tiene 63.000 estudiantes. Universidad Nacional Autónoma de México UNAM; crisis 2000 -2003. En la actualidad la UNAM tiene más de 300.000 estudiantes y más de 20,000 profesores de tiempo completo.

(3) La Constitución colombiana establece en su artículo tercero, que el constituyente primario es el pueblo y que alli reside su soberanía. Dentro de la misma lógica, el constituyente primario de una universidad es la Asamblea General Universitaria.

(4) Técnica de trabajo social que permite concertar visiones, estrategias y acciones para la construcción colectiva de escenarios futuros.

(5) Tünermann Carlos, La educación superior en el tercer milenio. UNESCO. Caracas Venezuela 1999.

(6) Órgano de poder decisorio universitario que en la actualidad es regulado por la ley 30 de 1992.

(7) Concepto profundizado por Max Weber para describir las formas de organización de las instituciones en las sociedades modernas.

(8) Figura normativa burocrática que le permite al titular de una representación ante un organismo de decisión enviar su reemplazo con variadas capacidades de negociación o de presencia.

(9) Figura normativa burocrática que le permite a los HCSU y a otras organizaciones maniobrar intereses que pueden acercarse en la toma de decisiones al equilibrio o a la arbitrariedad.

(10) El lector óptico-onda ha desordenado desde las nuevas versiones de una música de alta complejidad electrónica, que recrea las anteriores y puede ser creada al gusto de un solo actor que modula sus gustos, pasiones y desviaciones musicales, que puede ahora crear si así lo desea e incluso formar parte de la creación desde una dimensión próxima virtual.

(11) Gabiña Juanjo, El futuro revisitado. La reflexión prospectiva como arma de estrategia de decisión Alfomega Marcombo.

(12) Didriksson T Axel. Serie de conferencias sobre la universidad del futuro y sus textos sobre el mismo tema, Centro de estudios Universitarios CESU, UNAM, México, México 2001.

\section{BIBLIOGRAFÍA}

1. Didriksson T. Axel. Serie de conferencias sobre la universidad del futuro y sus textos sobre el mismo tema, Centro de estudios Universitarios CESU, UNAM, México, México 2001.

2. Gabiña Juanjo, El futuro revisitado. La reflexión prospectiva como arma de estrategia de decisión Alfomega Marcombo.

3. Machado, Ana Luisa, Directora OREALC UNESCO, Ana Maria Corvalán Coordinadora SIRI OREALC. "La educación en América Latina y el Caribe Prospectiva al 2020. Rio de Janeiro 1999.

4. Tünermann Carlos, La educación superior en el tercer milenio. UNESCO. Caracas, Venezuela 1999. 